\title{
La dirección escolar ante la participación del alumnado ${ }^{1}$
}

\section{School principals' point of view on pupils' participation}

\author{
LAURA García-RaGa*2 \\ laura.garcia@uv.es \\ Maria Carme Boqué Torremorell** \\ mariacarmebt@blanquerna.url.edu \\ Montserrat Alguacil de Nicolás** \\ montserratan@blanquerna.url.edu \\ * Universidad de Valencia, España \\ **Universitat Ramon Lull, España
}

\section{Resumen:}

La participación es un derecho que los niños y niñas deben tener la oportunidad de ejercer y la escuela es un escenario idóneo para que el alumnado pueda hacer oír su voz exponiendo ideas, tomando decisiones y formulando propuestas. Con el objetivo de detectar las opciones del alumnado de participar en el centro y apuntar vías para maximizarlas efectuamos un estudio a partir de un cuestionario elaborado ad hoc dirigido a todos y todas los directores y directoras de escuelas de Cataluña. En concreto, participaron 684 y los resultados indican que los niños y las niñas no solo opinan en clase, sino que toman decisiones cuando trabajan en grupo, en relación con la convivencia o votando sus propias propuestas. Además, pueden influir en el centro reivindicando y defendiendo sus intereses. En conclusión, los diferentes grados de participación del ción de los estudiantes.

\begin{abstract}
:
Participation is a right that children should have the opportunity to put into practice. Schools are an ideal setting for students to make their voices heard by expressing ideas, making decisions and formulating proposals. With the aim of exploring the options of the students to participate in the school and to point out ways to maximize these options, we conducted a study by means of an ad-hoc questionnaire addressed to all school principals in Catalonia. Specifically, 684 participated and the results indicate that not only do boys and girls give their opinion in class, but also make decisions when they work in groups, in relation to classroom coexistence or by voting their own proposals. In addition, they can influence the school by claiming and defending their interests. In conclusion, the different degrees of
\end{abstract}

1 Esta investigación se ha llevado a cabo gracias a la subvención obtenida en la convocatoria ORDRE GRI/183/2015, de 10 de juny, per la qual s'aproven les bases reguladores per a la concessió d'ajuts a projectes de recerca en l'àmbit de la qualitat democràtica i s'obre la convocatòria per a l'any 2015. DOGC Núm. 6897 - 22.6.2015 (Ref. 2015democ00016).

2 Dirección para correspondencia (correspondence address):

Laura García-Raga. Dirección: Avda/Blasco Ibañez, 30, 46010 Valencia (España). 
alumnado revelan una evolución desde la participación simbólica o aparente hacia opinar y decidir y, también, incidir. Sin embargo, la participación en los ámbitos tradicionalmente regidos por los adultos y la posibilidad de tomar iniciativas a la hora de formular los propios mecanismos participativos son poco frecuentes. Convendría, pues, fortalecer los diferentes niveles de participación y promover aquellos tipos que apenas se han detectado en este estudio.

\section{Palabras clave:}

Democracia; derechos del niño y la niña; director y directora del centro; participa- student participation reveal an evolution from symbolic or apparent participation towards giving opinions and making decisions, an also having an influence. However, participation in areas traditionally governed by adults and the possibility of establishing participatory mechanisms are rare. It would therefore be appropriate to strengthen the different levels of participation and to promote those types that have hardly been detected in this study.

\section{Keywords:}

Democracy; rights of the child; headteacher; student participation.

\section{Résumé:}

La participation est un droit que les enfants doivent avoir la possibilité d'exercer et l'école est un cadre idéal pour que les élèves fassent entendre leur voix en avançant des idées, en prenant des décisions et en formulant des propositions. Dans le but de détecter les options de participation des élèves à l'école et d'indiquer les moyens de les maximiser, nous avons réalisé une étude basée sur un questionnaire préparé ad hoc et adressé à tous les directeurs d'école de Catalogne. Concrètement, ils ont participé 684 et les résultats indiquent que les enfants ne donnent pas seulement leur avis en classe, mais qu'ils prennent également des décisions lorsqu'ils travaillent en groupe, en relation avec la coexistence ou en votant sur leurs propres propositions. En outre, ils peuvent influencer l'école en revendiquant et en défendant leurs intérêts. En conclusion, les différents degrés de participation des étudiants révèlent une évolution de la participation symbolique ou apparente vers l'expression d'opinions et la prise de décisions, ainsi que I'influence. Cependant, la participation dans les domaines traditionnellement gouvernés par les adultes et la possibilité de prendre des initiatives pour formuler leurs propres mécanismes participatifs sont peu fréquentes. II serait donc utile de renforcer les différents niveaux de participation et de promouvoir les types qui n'ont guère été détectés dans cette étude.

\section{Mots clés:}

Démocratie; droits de l'enfant; directeur et directrice de l'école; participation des élèves.

Fecha de recepción: 15-01-2020

Fecha de aceptación: 08-03-2020

\section{Introducción}

La construcción de sociedades inclusivas, innovadoras y reflexivas es uno de los retos prioritarios del programa Horizonte 2020, el cual impulsa acciones como: (a) promover organizaciones, prácticas, servicios 
y políticas fiables necesarias para construir sociedades inclusivas, participativas, abiertas; (b) explorar nuevas formas de innovación, con insistencia particular en la innovación y la creatividad sociales, y entender la manera en que todas las formas de innovación se desarrollan, alcanzan sus metas o fracasan; $y$, muy especialmente, (c) aprovechar el potencial innovador, creativo y productivo de todas las generaciones. Constatamos, sin embargo, que una parte significativa de la ciudadanía mundial suele ser sistemáticamente ignorada en los procesos democráticos de participación social, renunciando, así, a su potencial creativo y dejando sin voz a un buen número de personas con opinión propia. Nos referimos al colectivo infantil y adolescente.

Ahora bien, en el documento del Consejo de Europa (2012) titulado Recommendation on the participation of children and young under the age of 18, se pide a los países miembros que maximicen las oportunidades de la infancia de participar en aquello que le afecta y entre las medidas que señala figuran: el fomento de la participación activa de los niños y las niñas en la gobernanza de la escuela y el establecimiento de cuerpos consultivos en los ámbitos local, regional y nacional. No obstante, en muchos países, la participación infantil es un tema marginal que se encuentra fuera, incluso, de las agendas de transformación y cambio social que impulsan las entidades ciudadanas. Este hecho muestra el débil reconocimiento de niños y niñas como sujetos con derechos y alienta a unir esfuerzos en la construcción de una sociedad capaz de reconocer e incluir el protagonismo infantil en sus procesos de transformación y mejora (Santibáñez, Delano y Reyes, 2015).

Por otra parte, hay autores que han denunciado la infravaloración infantil en términos de subordinación, dependencia, necesidad de protección, insuficiente preparación, falta de madurez evolutiva, psicológica y moral, ciudadanía aplazada, negación jurídica, social, económica, cultural, etc., contribuyendo a reconocer sus capacidades y rol protagónico (Cussiánovich, 2003, 2010; Cussiánovich y Figueroa, 2009; Gaitán, 2006, 2008 y Liebel, 2000, 2007, 2013). En consecuencia, como nos dicen Espert y Boqué (2008), deben vencerse los prejuicios y las concepciones erróneas sobre los niños y las niñas para redescubrirlos como seres válidos, lejos de miradas sobreprotectoras, románticas o devaluadoras para devolverles, así, el rol protagónico que les corresponde.

En el contexto escolar, Howard y Wieczorek (2011, p. 2) afirman que la participación de los niños y las niñas en la gobernanza de la escue- 
la reporta una serie de beneficios: incrementa la autoestima, la autoconfianza y la creencia en las propias habilidades para generar cambio (empoderamiento); mejora las habilidades comunicativas y de escucha (competencias socio-personales); proporciona sentido de responsabilidad, acrecienta las habilidades y conocimientos sobre la democracia y la política (ciudadanía activa); disminuye el acoso y el aislamiento favoreciendo la interacción positiva entre iguales, entre niños, niñas y personas adultas y entre personas adultas (convivencia positiva); y se respetan más las decisiones cuando se les ha implicado en el proceso (pertenencia).Por otra parte, el impacto de la participación se hace sentir en la mejora de los resultados académicos y del aprendizaje, en un clima de convivencia más positivo y en las buenas relaciones entre los miembros de los diferentes sectores del centro, mejor comportamiento y disciplina, aumento de la confianza de los niños hacia el propio aprendizaje, mejoras en las prácticas docentes o la percepción de mayor legitimidad a las decisiones que se toman conjuntamente.

Ahora bien, como se dice en el informe de la UNICEF (2003) correspondiente al estado de la infancia en el mundo, la participación infantil no siempre es activa, social, significativa o constructiva y recalca que los niños y las niñas necesitan información, apoyo y condiciones favorables para participar de manera adecuada y digna. De cualquier forma, pueden identificarse diferentes grados de participación de los niños y las niñas en la escuela, los cuales se reflejan en la tabla 1a partir de las clasificaciones realizadas por diversos autores.

Tabla 1

Grados de participación infantil en la escuela

\begin{tabular}{ll}
\hline AUTORES & GRADOS DE PARTICIPACIÓN \\
\hline Arnstein & Manipulación, (1) terapia, (2) información, (3) consulta, (4) \\
(1969) & conciliación, (5) colaboración, (5) delegación de poder y (6) \\
& Control ciudadano. \\
Hart (1993) & -No participación: (1) manipulación, (2) decoración y (3) toke- \\
& nismo (aparente o simbólica). \\
& -Participación: (4) asignados pero informados, (5) consultados \\
& e informados, (6) decisiones iniciales de los adultos, compar- \\
& tidas por los niños, (7) decisiones iniciales y dirección de los \\
& niños y niñas y (8) decisiones iniciales de los niños y niñas, \\
& compartidas por los adultos. \\
Franklin (1995) & No-participación, pre-participación y participación.
\end{tabular}




\begin{tabular}{|c|c|}
\hline AUTORES & GRADOS DE PARTICIPACIÓN \\
\hline $\begin{array}{l}\text { Treseder } \\
(1997)\end{array}$ & $\begin{array}{l}\text { Hace hincapié en el poder, la toma de decisiones y la ejecu- } \\
\text { ción de los proyectos. }\end{array}$ \\
\hline $\begin{array}{l}\text { Mason y Urqu- } \\
\text { hart (2001) }\end{array}$ & $\begin{array}{l}\text { Distinguen entre modelos adultocéntricos, donde los adultos } \\
\text { marcan los límites de la participación, y modelos de participa- } \\
\text { ción dominada por los niños y las niñas, donde son ellos quie- } \\
\text { nes establecen la agenda; en el punto medio hallamos mode- } \\
\text { los colaborativos, donde los adultos ejercen el liderazgo, pero } \\
\text { asisten a los niños y las niñas para que puedan hacer oír su voz. }\end{array}$ \\
\hline Chawla (2001) & $\begin{array}{l}\text { Asignada, invitada, negociada, autoiniciada y negociada, en } \\
\text { grado y colaborativa. }\end{array}$ \\
\hline $\begin{array}{l}\text { Thomson y } \\
\text { Holdsworth } \\
\text { (2003) }\end{array}$ & $\begin{array}{l}\text { Establecen el siguiente continuum: estar físicamente pre- } \\
\text { sente; implicarse tomando parte en las clases y actividades; } \\
\text { participar informalmente en la toma de decisiones (consul- } \\
\text { tas, comités); iniciar, decidir y actuar en la escuela y más allá } \\
\text { (capacidad de construir vecindario); y organizar proyectos } \\
\text { comunitarios y de activismo social (defensa de los derechos } \\
\text { humanos, protestas contra injusticias, etc.). Se observa, por } \\
\text { tanto, una clara evolución en todas las clasificaciones expues- } \\
\text { tas, desde aceptar, opinar y decidir hasta incidir o reivindicar. }\end{array}$ \\
\hline Mitra (2007) & $\begin{array}{l}\text { Gradúa la participación según si los adultos escuchan a la } \\
\text { infancia, si hay colaboración niño-adulto o bien si los niños y } \\
\text { las niñas lideran los procesos de cambio }\end{array}$ \\
\hline
\end{tabular}

Trilla y Novella Proponen Cuatro tipologías de participación: simple, consul(2001) y Nove- tiva, proyectiva y metaparticipación.

Ila (2008)

Lundy (2007) Este modelo adoptado en países como Irlanda, comprende cuatro elementos que siguen un orden cronológico: espacio, voz, audiencia e influencia.

Nolan (2015) Considera que se debe replantear la participación infantil como un tipo de ciudadanía cuyas formas de acción no siempre se reconocen y hacer visibles sus conexiones con el activismo, la ciudadanía y el cambio social.

Herbots y Put La participación infantil parece ser un concepto "container" y (2015) recalca que participación no es sinónimo de inclusión. En su disco de la participación incluyen: modo, propósito, contexto y agentes involucrados.

Gal (2017) Promueve un modelo ecológico de participación infantil con los siguientes elementos: condiciones predeterminadas, contexto, resultados individuales y resultados estructurales.

Tisdall y Punch Cuestionan la participación infantil entendida acríticamente (2018) como un "mantra" y defienden que debe dar paso a estudios más profundos y problematizadores sobre el rol social y agente de la infancia.

Nota: Elaboración propia a partir de las clasificaciones ofrecidas por autores. 
Haciendo una síntesis de las diferentes categorizaciones, en el trabajo presentado haremos alusión a cuatro grados de participación: aceptar como sujeto pasivo tomar parte en una propuesta ya organizada (A); opinar, es decir, expresar el propio punto de vista sobre cuestiones diversas $(\mathrm{O})$; decidir, consistente en elegir una vía de acción de acuerdo con los propios intereses (D); e incidir, que se refiere a intervenir significativamente en el funcionamiento del centro (I). El primer grado de participación se refiere a una participación restringida o simulada, mientras que las tres últimas se vinculan con una participación más genuina. Se tratará de analizar los grados de participación y los espacios de participación infantil más extendidos en las escuelas de infantil y primaria de Cataluña. Para ello, se consideran las percepciones de la dirección escolar de los centros participantes en este estudio para, finalmente, formular algunas recomendaciones que permitan maximizar el rol protagónico de la infancia en la escuela infantil y primaria. Nos interesa conocer cómo entienden la participación infantil en la escuela los directores y las directoras, ya que tienen una visión de conjunto tanto en el ámbito organizativo, como en el pedagógico o en el social. Son, además, los impulsores de las políticas del centro y responsables de garantizar la calidad y la equidad educativa. Es a este colectivo al que hemos dirigido cuestiones fundamentales referentes al alcance y el grado en que se hace efectiva la participación en su centro y al acceso que tiene el alumnado a tomar parte en la gobernanza del centro. Obviamente, se trata tan sólo de una percepción subjetiva, pero que, en su conjunto, ofrece un retrato bastante nítido del estado general de la participación infantil en las escuelas de Cataluña.

\section{Diseño y metodología}

Se trata de un diseño basado en un estudio de encuesta desde una perspectiva cuantitativa, con el objetivo de conocer las percepciones de la dirección escolar sobre la participación del alumnado de Educación Infantil y Primaria en sus centros educativos. El contexto de la investigación es la Comunidad Autónoma de Cataluña, respondiendo a una convocatoria competitiva de dicha comunidad y tomando este territorio como población inicial de un estudio a replicar en el resto del Estado. 


\section{Objetivos}

Como objetivo general, el estudio pretende conocer el alcance de la participación del alumnado en centros educativos de Cataluña a partir de las percepciones de la dirección escolar. Este objetivo se concreta en los siguientes más específicos:

- Detectar las prácticas participativas del alumnado más frecuentes en sus centros educativos.

- Analizar los grados de participación del alumnado en los centros educativos desde la perspectiva de la dirección escolar.

- Descubrir diferencias en los grados de participación infantil de acuerdo con las direcciones escolares.

\section{Población y muestra}

La población está constituida por todos los directores y directoras de los centros de Educación Infantil y Primaria de Cataluña, en total 2,378. A todos ellos se les envió cuestionario, si bien decidieron responder $y$, por tanto, participar en el estudio 684 directores y directoras. La muestra, de esta manera, supera el tamaño que se considera como representativo de la población (331 sujetos con un nivel de confianza del 95\% y 5\% de margen de error).

La tabla 2 presenta los centros de los directores y directoras de la muestra según su titularidad. Respecto al número de alumnado, el $83 \%$ de los centros educativos tienen menos de 500 estudiantes y el $17 \%$ entre 500 y 3,000 .

Tabla 2

Titularidad de los centros de la muestra

\begin{tabular}{lcc}
\hline Titularidad & Frecuencia & Porcentaje \\
\hline Centro concertado - confesional & 99 & 14.5 \\
Centro concertado - laico & 52 & 7.6 \\
Centro público - Generalitat de Catalunya & 512 & 74.9 \\
Centro público - municipal & 15 & 2.2 \\
Centro privado - cooperativa & 3 & .4 \\
Centro privado - no cooperativa & 3 & .4 \\
Total & 684 & 100 \\
\hline
\end{tabular}




\section{Instrumento}

Para el logro de los objetivos planteados se elaboró un cuestionario ad hoc, considerando las siguientes fases: a) abordaje teórico y conceptual de la participación infantil en los centros educativos; b) diseño del instrumento; c) validación por grupo investigador y jueces.

Así pues, analizado el contenido de la propuesta inicial del instrumento (validez de constructo) por el grupo de investigación, fue sometido al juicio de 6 expertos tanto en metodología como en participación escolar, en función de los criterios de univocidad, pertinencia e importancia (validación lógica). A partir de las valoraciones aportadas e indicaciones de los evaluadores se ha confeccionado el instrumento final. El cuestionario cuenta con 17 ítems, si bien nos vamos a centrar en este artículo únicamente en 5, los cuales están referidos a los grados de participación del alumnado. Los ítems son cerrados y cuentan con 4 opciones, ofreciéndose espacio para "otras" respuestas. Las respuestas de los ítems han sido categorizadas en función del grado de participación: aceptar como sujeto pasivo tomar parte en una propuesta ya organizada (A); opinar, es decir, expresar el propio punto de vista sobre cuestiones diversas $(\mathrm{O})$; decidir, consistente en elegir una vía de acción de acuerdo con los propios intereses (D); e incidir,que se refiere a intervenir significativamente en el funcionamiento del centro (I).

\section{Procedimiento}

El cuestionario se envió on-line a todos los directores y a las directoras de centros educativos de Cataluña de infantil y primaria (formulario de Google), acompañado de una carta de apoyo del Consejo Escolar de Cataluña, máximo órgano promotor de la participación en la comunidad educativa. La carta valoraba la oportunidad del estudio, el interés de la temática y el acierto de la metodología utilizada. El procedimiento de análisis cuantitativo de datos se ha efectuado con el paquete estadístico Statistical Package for the Social Sciences (SPSS) versión 24, realizándose un análisis descriptivo de los datos recogidos del cuestionario para conocer la opinión y valoración que ofrece la dirección en lo referente a la participación del alumnado. Además, cabe indicar que dos jueces independientes han categorizado analíticamente las respuestas a partir de un sistema de doble ciego. 


\section{Resultados}

Una vez analizados los datos, pasamos a exponer los resultados, organizándolos en función de las respuestas ofrecidas a cada pregunta y relacionándolos con los cuatro grados de participación ya señalados: A (aceptar), O (opinar), D (decidir), I (incidir). Posteriormente a cada tabla, sintetizamos los grados de participación gráficamente. Cabe indicar que, en ocasiones, las respuestas son mayores a la muestra porque algunos directores o directoras incluían más de una respuesta en la opción "otras".

En cuanto a la primera pregunta, "En nuestra escuela, las actividades más habituales en las que participan los niños y las niñas son...", se obtuvieron respuestas que se pueden categorizar en los distintos grados de participación, taly como se observa en la tabla 3, además de recabarse abundantes respuestas en el apartado "otras".

Tabla 3

Presencia participativa del alumnado a través de su voz

Frecuencia Porcentaje $\begin{gathered}\text { Grado de } \\ \text { participación }\end{gathered}$

En nuestra clase, las actividades más ha-

bituales en las que participan los niños y

las niñas son...

\begin{tabular}{|c|c|c|c|c|}
\hline a. Opinar & en clase & 250 & 36.54 & $\mathrm{O}$ \\
\hline b. Interve & nir en la solución de conflictos & 175 & 25.58 & $\mathrm{D}$ \\
\hline c. Evalua & r el funcionamiento de la clase & 90 & 13.15 & I \\
\hline d. Prepar & ar una fiesta & 44 & 6.43 & A \\
\hline e. Otras & $\begin{array}{l}\text { Asambleas de aula, de delega- } \\
\text { dos y de alumnos; reuniones } \\
\text { de delegados y equipo direc- } \\
\text { tivo para tratar: mediación de } \\
\text { conflictos, actividades de aula, } \\
\text { reformas o mejoras en la es- } \\
\text { cuela, organización de fiestas } \\
\text { y otros temas del centro }\end{array}$ & 27 & 3.95 & I \\
\hline & $\begin{array}{l}\text { Comités, consejos y comisio- } \\
\text { nes }\end{array}$ & 24 & 3.50 & I \\
\hline & $\begin{array}{l}\text { Opinar en diferentes aspectos } \\
\text { de la escuela e intervenir en la } \\
\text { resolución de conflictos }\end{array}$ & 17 & 2.48 & $\mathrm{O}$ \\
\hline & $\begin{array}{l}\text { Responsabilizarse en las mejo- } \\
\text { ras que propone el centro }\end{array}$ & 12 & 1.75 & A \\
\hline
\end{tabular}




\begin{tabular}{|c|c|c|c|}
\hline & Frecuencia & Porcentaje & $\begin{array}{c}\text { Grado de } \\
\text { participación }\end{array}$ \\
\hline $\begin{array}{l}\text { Organizar actividades de la } \\
\text { escuela }\end{array}$ & 12 & 1.75 & I \\
\hline Aportar ideas y soluciones & 7 & 1.02 & $\mathrm{O}$ \\
\hline $\begin{array}{l}\text { Escoger proyectos de aula o de } \\
\text { escuela }\end{array}$ & 6 & .88 & $\mathrm{D}$ \\
\hline Intervenir en todos los ámbitos & 6 & .88 & I \\
\hline $\begin{array}{l}\text { Evaluar el funcionamiento del } \\
\text { aula y de la escuela }\end{array}$ & 5 & .73 & I \\
\hline $\begin{array}{l}\text { Gestionar el grupo clase y la } \\
\text { escuela }\end{array}$ & 4 & .58 & I \\
\hline $\begin{array}{l}\text { Realizar propuestas para tota } \\
\text { la comunidad educativa }\end{array}$ & 1 & .15 & I \\
\hline $\begin{array}{l}\text { Decidir sobre aquello que } \\
\text { quieren aprender }\end{array}$ & 1 & .15 & $\mathrm{D}$ \\
\hline $\begin{array}{l}\text { Intervenir en la gobernanza de } \\
\text { la escuela }\end{array}$ & 1 & .15 & I \\
\hline $\begin{array}{l}\text { Tomar la palabra en las sesio- } \\
\text { nes de clase }\end{array}$ & 1 & .15 & $\mathrm{O}$ \\
\hline Total & 684 & 100 & \\
\hline
\end{tabular}

Se detecta que las tres cuartas partes de las respuestas se agrupan en torno a las opciones dadas, quedando una cuarta parte (124 respuestas) de comentarios que matizan, precisan o formulan nuevas acciones.

En la figura 1 se representan los diferentes grados de participación detectados que, en general, parecen inclinarse por niveles de participación real en la escuela. Como puede verse, predomina la posibilidad de opinar en clase, siendo menor el grado de "aceptar", elegido minoritariamente $(8.18 \%)$. Además, cuando entramos en el terreno de los adultos y preguntamos por la evaluación del funcionamiento del aula, el número de respuestas disminuye (13.15\%). Pero si, además, se consideran sus aportaciones según las "otras" respuestas,el $26.61 \%$ de las respuestas se agrupan bajo el grado de decidir y el $24.84 \%$ en el de incidir. 


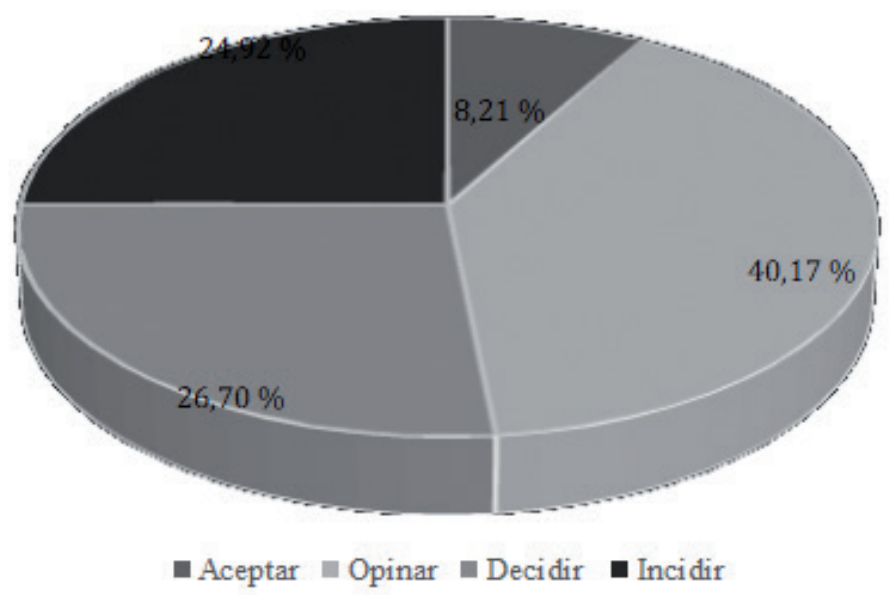

Figura 1. Gráfico de los grados de participación del alumnado en cuanto a la presencia participativa del alumnado a partir de su voz.

En la siguiente pregunta, “En nuestra escuela, los niños y las niñas también suelen participar en...", insistimos sobre las opciones de participación del alumnado en la escuela a través de su voz, dando nuevamente a elegir entre diferentes actividades. Los resultados aparecen en la tabla 4. Así pues, el $89.86 \%$ priorizó las opciones propuestas, donde "elaborar normas de convivencia" sobrepasó la mitad de las respuestas (53.34\%). En segundo lugar, los niños y las niñas participan en cuestiones referentes a los contenidos de aprendizaje (23.48\%) y de manera más minoritaria colaboran en campañas organizadas por la escuela $(9.42 \%)$ y sólo un $3.62 \%$ participa en la evaluación de los docentes. Por otra parte, cada vez hay más centros que trabajan mediante proyectos, grupos cooperativos, aprendizaje y servicio, entre otras aproximaciones didácticas que dan voz al alumnado frente al aprendizaje, si bien, como se observa aquí, las propuestas arrancan del docente. En el apartado "otras", algunas respuestas señalan explícitamente que el alumnado decide los temas de trabajo, pero son minoritarias. Asimismo, se señala la colaboración en campañas solidarias poniendo de relieve la dimensión social de la escuela y su proyección al entorno. 
Tabla 4

Presencia participativa del alumnado a partir de su voz en otras actividades

Grado

Frecuencia Porcentaje de parti-

cipación

1. En nuestra escuela, los niños y las niñas

también suelen participar en...

a. Elaborar normas de convivencia (D)

b. Elegir entre algunos temas de estudio que propone el docente $(\mathrm{O})$

c. Reunir dinero para una causa o campaña organizada por la escuela (A)

d. Evaluar a los docentes (I)

e. Otras a) Proponer fiestas, celebraciones, patio, etc.

b) Participar en proyectos curriculares, transversales entre ciclos, plásticos, etc. organizados por el equipo docente

c) Escoger temas de estudio

d) Participar en asambleas (delegados, patio)

e) Elegir proyectos de trabajo

f) Miembros de comisiones de trabajo, comités (ejemplo: escuela verde), campañas solidarias, etc.

g) Demandas e iniciativas del alumnado

h) Aportaciones al Consejo de la Infancia y al Consejo de Delegados

i) Acompañar a otros compañeros en el aprendizaje a petición del docente

j) Evaluar el proceso de aprendizaje y las actividades del curso

k) Gestionar el grupo

I) Participar en el proceso de enseñanza- aprendizaje y en todo lo que les atañe

m) Escoger representantes

n) Formular preguntas

o) Gestión y organización del centro Total

\begin{tabular}{|c|c|c|}
\hline 368 & 53.34 & D \\
\hline 162 & 23.48 & $\mathrm{O}$ \\
\hline 65 & 9.42 & A \\
\hline 25 & 3.62 & I \\
\hline 13 & 1.88 & I \\
\hline 11 & 1.59 & A \\
\hline 8 & 1.16 & $\mathrm{D}$ \\
\hline 7 & 1.01 & I \\
\hline 6 & .87 & D \\
\hline 5 & .72 & I \\
\hline 4 & .58 & I \\
\hline 3 & .43 & I \\
\hline 4 & .58 & A \\
\hline 2 & .29 & I \\
\hline 2 & .29 & I \\
\hline 2 & .29 & I \\
\hline 1 & .14 & D \\
\hline 1 & .14 & $\mathrm{O}$ \\
\hline 1 & .14 & I \\
\hline 690 & 99.97 & \\
\hline
\end{tabular}


En la figura 2 reflejamos los grados de participación en función de las respuestas anteriores.

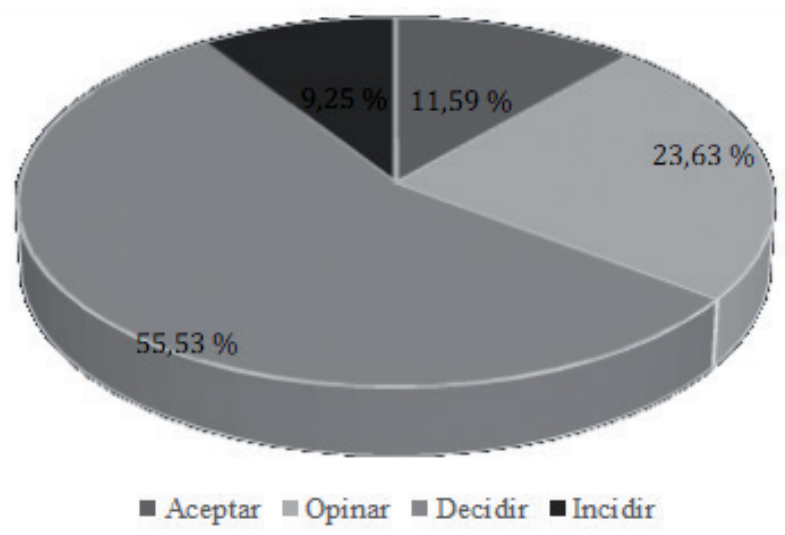

Figura 2. Gráfico de los grados de participación del alumnado en cuanto a la presencia participativa del alumnado a partir de su voz en otras actividades.

En cuanto a la tercera pregunta formulada como "En nuestra escuela, los niños y las niñas pueden...", se pretendía conocer de qué manera el alumnado participa en toma de decisiones (tabla 5). Aquí se destaca que en cuanto a "reivindicar un derecho y defender los propios intere-

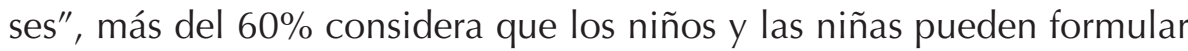
quejas y peticiones de acuerdo con las percepciones de la dirección escolar. Con un nivel de respuesta mucho menor, no obstante, se indica que el alumnado asiste a las entrevistas de los docentes con las familias y, todavía en menor proporción, participa en la selección de materiales didácticos o excursiones propuestas por los docentes. Asimismo, resultan interesantes los múltiples comentarios añadidos vinculados con las preguntas anteriores $(b, c, e, f, h, i, j, k, l, s)$ o que ejemplifican prácticas particulares $(a, d, g, m, o, p, q, r)$. 
Tabla 5

Presencia participativa del alumnado a partir de su toma de decisiones

Frecuencia Porcentaje de participación

1. En nuestra escuela, los niños y las niñas pueden...

a. Reivindicar un derecho y defender los pro- 440 pios intereses (I)

63.76

b. Participar en las entrevistas entre docente y 99 familia (D)

c. Intervenir en la elección de materiales didác- 46 ticos y/o libros (D)

d. Elegir las excursiones entre las que propone 25

el equipo docente $(\mathrm{O})$

a) Organizar juegos, celebraciones, fiestas, colonias, proyectos, materiales, espacios o talleres de comedor

b) Proponer y/o decidir temas de interés $y$ estudio

c) Intervenir en la organización del centro y proponer mejoras

d) Exponer su criterio u opinión

e) Elegir proyectos de aula

f) Hacer propuestas al Consejo de alumnos, asambleas, tutorías, consistorio infantil, comité verde, etc.

\begin{tabular}{|c|c|c|}
\hline g) Proponer actividades del centro & 5 & .72 \\
\hline $\begin{array}{l}\text { Th) Participar activamente en su aprendizaje } \\
\text { i/o enresponsables de cargos del aula }\end{array}$ & 4 & .58 \\
\hline${ }^{\top}$ i) Plantear soluciones a los conflictos & 4 & .58 \\
\hline j) Decidir normas de aula/escuela & 3 & .43 \\
\hline $\begin{array}{l}\text { k) Participar en actividades propuestas por el } \\
\text { centro/tutores }\end{array}$ & 2 & .29 \\
\hline I) Gestionar el aula & 2 & .29 \\
\hline $\begin{array}{l}\text { m) Ninguna de las indicadas en el cuestio- } \\
\text { nario }\end{array}$ & 2 & .29 \\
\hline $\begin{array}{l}\text { n) Crear actividades para el resto de alum- } \\
\text { nado }\end{array}$ & 1 & 14 \\
\hline o) Decidir inversiones del centro & 1 & 14 \\
\hline p) Préstamo de libros & 1 & 14 \\
\hline $\begin{array}{l}\text { q) Aprendizaje y servicio organizado por el } \\
\text { centro }\end{array}$ & 1 & .14 \\
\hline
\end{tabular}




\begin{tabular}{lccc}
\hline & Frecuencia Porcentaje $\begin{array}{c}\text { Grado } \\
\text { de parti- } \\
\text { cipación }\end{array}$ \\
\hline $\begin{array}{l}\text { r) Reivindicar derechos siendo responsables } \\
\text { de sus deberes }\end{array}$ & 1 & .14 & I \\
Total & 690 & 99.22 & \\
\hline
\end{tabular}

En la figura 3 se muestran los grados de participación referidos a la toma de decisiones del alumnado, destacándose, como puede verse, el grado de incidir aunque desigualmente representado, puesto que se reconoce que el alumnado tiene voz para reclamar sus derechos $(63.76 \%)$ y en porcentajes muy menores se ven reflejadas opciones que, de algún modo, implican entrar en el terreno de decisión docente: organización de juegos, celebraciones, etc. (2.17\%), proponer y/o decidir temas de estudio (1.45\%), intervenir en la organización del centro $(1.30 \%)$ y otras opciones que alcanzan un porcentaje inferior al $1 \%$.

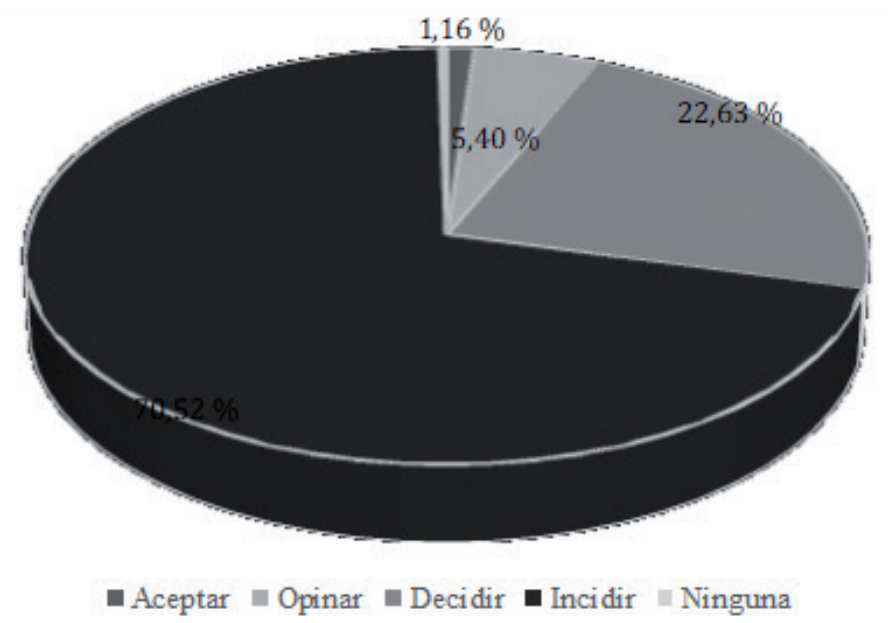

Figura 3. Gráfico de los grados de participación del alumnado en cuanto a la toma de decisiones.

En cuanto a la cuarta pregunta, relativa a las prácticas de participación en el aula, los resultados se exponen en la tabla 6. Como se observa, la respuesta "trabajar colaborativamente" casi alcanza el 80\%, seguida por "proponer temas de estudio", próxima al 10\%, y por "organizar el trabajo del aula", con una puntuación que supera mínimamente el 5\%; de manera casi anecdótica, "asistir a las reuniones de evaluación", ob- 
tiene una única respuesta (.15\%). El apartado "otras", en esta ocasión, se sitúa alrededor del $7 \%$ y complementa las opciones dadas.

Tabla 6

Prácticas de participación del alumnado en el aula

Grado de

Frecuencia Porcentaje participación

4. En nuestra escuela, la manera como los niños y niñas participan en el aula consiste en...

\begin{tabular}{|c|c|c|c|}
\hline a. Trabajar colaborativamente & 548 & 79.76 & $\mathrm{D}$ \\
\hline $\begin{array}{l}\text { b. Proponer los temas de estudio que se tra- } \\
\text { bajan en clase }\end{array}$ & 66 & 9.60 & $\mathrm{D}$ \\
\hline c. Organizar el trabajo del aula & 40 & 5.82 & $\mathrm{D}$ \\
\hline d. Asistir a las reuniones de evaluación & 1 & .14 & 1 \\
\hline Asambleas de toma de decisiones & 6 & .87 & I \\
\hline Día a día del aula y aprendizajes & 6 & .87 & A \\
\hline Aprender cooperativamente & 5 & .73 & A \\
\hline Tutorías entre iguales & 3 & .44 & A \\
\hline $\begin{array}{l}\text { Asistencia a reuniones con voz y voto (ci- } \\
\approx \text { vismo, escuela verde, delegados) }\end{array}$ & 3 & .44 & I \\
\hline Resolución de conflictos entre pares & 3 & .44 & I \\
\hline${ }^{\top}$ Consejo de delegados & 2 & .29 & I \\
\hline Debates y conversaciones & 2 & .29 & $\mathrm{O}$ \\
\hline Opinar y votar & 1 & .15 & $\mathrm{D}$ \\
\hline Todo lo que se hace en la escuela & 1 & .15 & I \\
\hline Total & 687 & 99.55 & \\
\hline
\end{tabular}

En la figura 4, reflejamos los grados de participación en función de las respuestas obtenidas, destacando, como puede observarse, el grado de decidir. 


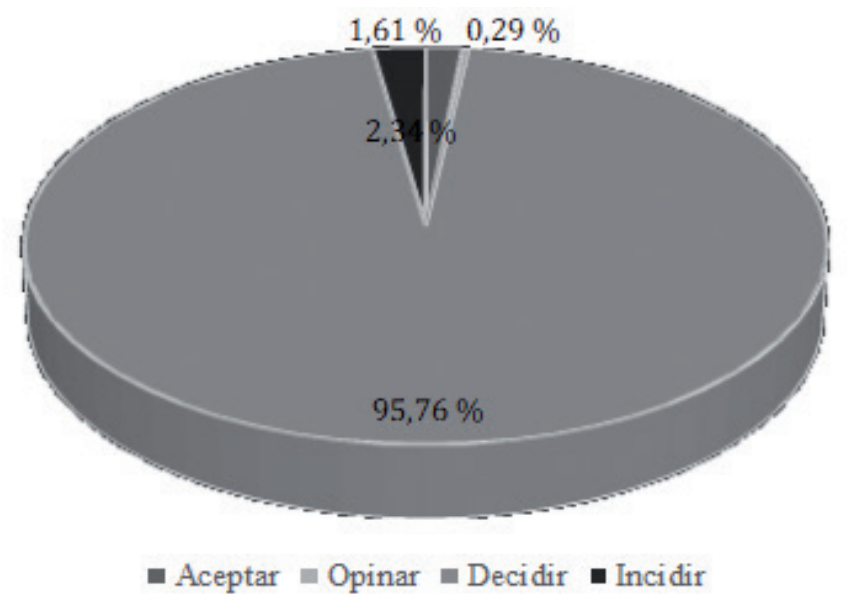

Figura 4. Gráfico de los grados de participación del alumnado en cuanto a las prácticas participativas del alumnado en su aula.

Finalmente, mostramos en la tabla 7 las respuestas a la pregunta sobre las prácticas de participación en el centro educativo. Como se detecta, "Votar las propuestas que formulan los compañeros" es la opción más marcada (41.98\%), indicando que el alumnado puede proponer y decidir. Por otra parte, resulta interesante la proporción de directores y directoras que afirman que el alumnado evalúa el funcionamiento del centro y puede tomar decisiones sobre aspectos organizativos, ya que ambas categorías se corresponden con incidir. En otros casos, el alumnado vota propuestas lideradas por el adulto.

Tabla 7

Prácticas de participación del alumnado en el centro educativo

Grado de

Frecuencia Porcentaje Participación

5. En nuestra escuela, la manera como los niños y niñas participan en el centro consiste en...

\begin{tabular}{lccc}
$\begin{array}{l}\text { a. Votar las propuestas que formulan los com- } \\
\text { pañeros y compañeras }\end{array}$ & 288 & 41.98 & D \\
$\begin{array}{l}\text { b. Evaluar el funcionamiento de la escuela } \\
\begin{array}{l}\text { c. Votar las propuestas que formula la escuela } \\
\text { d. Tomar parte en decisiones sobre aspectos }\end{array}\end{array}$ & 100 & 18.07 & I \\
$\begin{array}{l}\text { organizativos de la escuela: horarios, uso de } \\
\text { espacios, agrupamientos, etc. }\end{array}$ & 70 & 10.23 & I \\
\hline
\end{tabular}




\begin{tabular}{|c|c|c|c|}
\hline & Frecuencia & Porcentaje & $\begin{array}{l}\text { Grado de } \\
\text { Participa- } \\
\text { ción }\end{array}$ \\
\hline $\begin{array}{l}\text { a) Participar en asambleas, consejos y } \\
\text { diversas actividades de gestión del centro }\end{array}$ & 45 & 6.51 & I \\
\hline b) Hacer propuestas & 28 & 4.05 & I \\
\hline $\begin{array}{l}\text { c) Consensuar decisiones sobre diversos } \\
\text { aspectos del centro }\end{array}$ & 15 & 2.17 & I \\
\hline $\begin{array}{l}\text { d) Evaluar las actividades organizadas y } \\
\text { co-evaluar el aprendizaje }\end{array}$ & 5 & .72 & I \\
\hline $\begin{array}{l}\text { e) Ninguna de las opciones indicadas en } \\
\text { el cuestionario }\end{array}$ & 5 & .72 & --- \\
\hline f) Colaborar en el aula y en la escuela & 4 & .58 & A \\
\hline $\begin{array}{l}\approx \text { g) El alumnado no participa en la toma de } \\
\bigcirc \text { decisiones y no vota }\end{array}$ & 2 & .29 & A \\
\hline 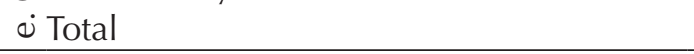 & 686 & 100.08 & \\
\hline
\end{tabular}

En la figura 5, mostramos, como con los demás ítems, los grados de participación.

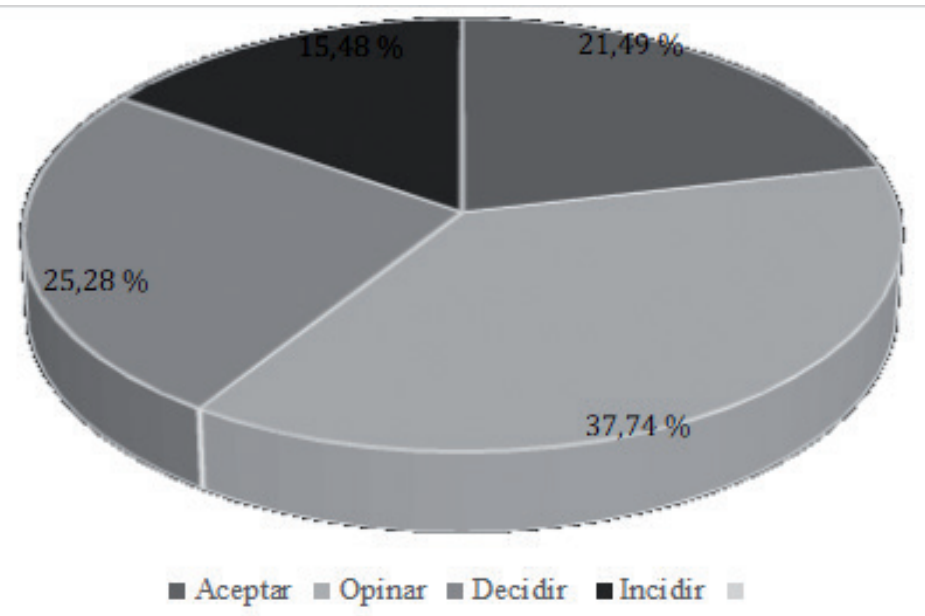

Figura 5. Gráfico de los grados de participación del alumnado en cuanto a las prácticas participativas del alumnado en sus centros educativos.

Habiendo analizado las respuestas de cada pregunta y vinculándolas con los grados de participación, se muestra, a modo de síntesis, los grados de participación reflejados en cada ítem, así como las puntuaciones promedio obtenidas (figuras 6 y 7 respectivamente). 
Como se observa, el grado de participación más frecuente según las percepciones de la dirección escolar es el de decidir, seguido por el de opinar, si bien sobre todo en cuestiones vinculadas con el aula (ítem 4).

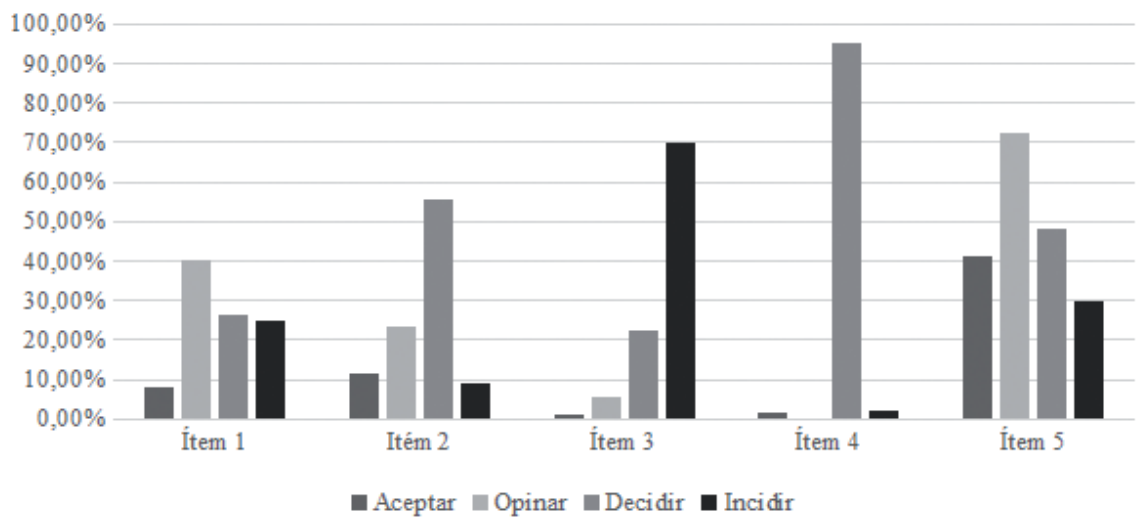

Figura 6. Gráfico de la síntesis de los grados de participación del alumnado por ítems.

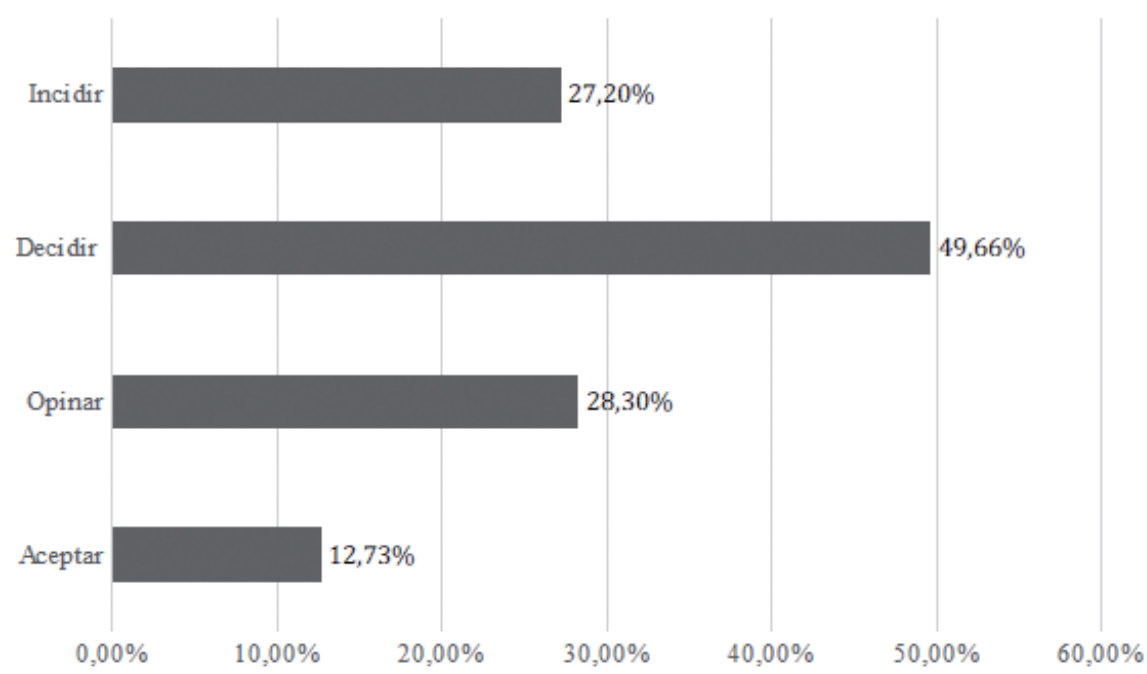

Figura 7. Gráfico del promedio de las respuestas de los directores y las directoras en cuanto a los grados de participación del alumnado. 


\section{Discusión y Conclusiones}

Conforme al objetivo general planteado en esta investigación, conocer la valoración que la dirección escolar de centros educativos de Cataluña otorga al alcance de la participación del alumnado, comenzamos subrayando que, según los resultados de este estudio, podemos decir que, en general, los centros de educación infantil y primaria de Cataluña promueven la participación con el alumnado, otorgándole un papel activo y protagonista, lo cual veíamos como fundamental, tal y como reclaman Alfageme, Cantos y Martínez (2003), Santibáñez et al. (2015), o Woodhouse (2003), entre otros autores.

Ahora bien, como mostraban los resultados señalados y respondiendo a nuestro objetivo de detectar las prácticas de participación más extendidas, cabe decir que la actividad más extendida en la escuela consiste en poner de manifiesto la propia opinión (36.54\%), seguida por la posibilidad de intervenir en la solución de sus conflictos (25.58\%). Esto nos indica que la verdadera participación infantil es todavía un aspecto que alcanzar en la escuela. Lo cierto es que la opción de incidir en el funcionamiento del centro aquí sólo la reconoce un $11.5 \%$ de los directores y directoras.

Sin embargo, cabe resaltar que más de las tres cuartas partes de las direcciones escolares señalan que los niñas y niñas deciden en aspectos de aprendizaje (trabajo colaborativo), convivencia (elaboración de normas) y funcionamiento (votación de propuestas), con lo que puede decirse que se confía en las capacidades del alumnado, tal y como ya destacaban otros estudios (Autor, 2008; Cussiánovich, 2003, 2010; Gaitán, 2006, 2008; Liebel, 2013 y UNICEF, 2003). También queda claro que el adulto muestra disposición para escuchar quejas y sugerencias reconociendo los derechos y la dignidad de todas las personas (Guichot, 2013; Lansdown, 2001). Y aunque opinar se corresponda con un estadio inferior de participación, Arnstein (1963), el primero en categorizar la participación infantil, mostró tres niveles por debajo de consulta, como ya se destacaba en la introducción: manipulación, terapia e información.

En cuanto a las modalidades de participación más extendidas en el aula, se destaca el trabajo cooperativo $(79.76 \%)$, con lo que podemos decir que a los centros educativos les interesa actualmente que los niños y niñas aprendan los unos con los otros En cuanto a la participación en normas de convivencia, otra de las formas de participación seña- 
ladas por más de la mitad de directores y directoras, hay que destacar que desde hace años los centros docentes trabajan por la mejora de la convivencia motivados, desgraciadamente, por manifestaciones de violencia graves como el acoso entre compañeros o bullying, entre otros. Muchos centros, así pues, son conocedores del programa de convivencia y mediación escolar del Departamento de Enseñanza (Departament d'Educació, 2007) o del portal de convivencia donde los centros disponen de guías y recursos para trabajar en este ámbito. No nos extraña, por tanto, que las respuestas relacionadas con la convivencia en el centro sean elegidas por un buen número de direcciones. Feito (2009), a su vez, encuentra indicativo de un centro democrático que haya una gestión compartida de la convivencia y Kränzl-Nagl y Zartler (2010) concluyen que la participación de los niños en la gestión de la convivencia mejora el clima del centro.

En cuanto a la posibilidad de elegir entre temas de estudio, señalada únicamente por el $9.60 \%$ de respuestas, cabe decir que entendemos que, si bien el alumnado puede opinar y decidir, son los adultos quienes plantean las opciones posibles.

Sí que nos resulta más sorprendente que se indique con un porcentaje elevado (63.76\%) que los niños y niñas pueden reivindicar un derecho y defender los propios intereses en la escuela. No obstante, según sólo el $17.25 \%$ de directores y directoras, pueden evaluar el centro e incidir en cuestiones organizativas (10.23\%). Este último porcentaje es bastante bajo si tenemos en cuenta que Howard y Wieczorek (2011) creen que se debe incorporar a los niños y a las niñas en decisiones sobre la propia educación, tales como planificación de horarios, metodologías didácticas, acción docente, sistemas de evaluación, selección de contenidos o revisión del currículo, ya que forman parte de los intereses de los niños y las niñas y afectan enormemente su día a día en la escuela.

Con todo, la lectura global de las respuestas nos indica que los niños y las niñas no sólo opinan en sus escuelas (28.30\%), sino que toman decisiones cuando trabajan en grupo en relación con la convivencia en el centro o cuando votan las propias propuestas. Y, además, la posibilidad de influir en el centro se hace patente cuando se afirma que los niños y las niñas pueden reivindicar y defender los propios intereses. Así pues, las diferentes tipologías o grados de participación infantil están presentes en la escuela de infantil y primaria, indicando una evolución desde la participación simbólica o aparente (minoritaria) hacia opinar y decidir 
(mayoritarias) e, incluso, incidir. Todo esto, sin embargo, sin inmiscuirse demasiado en terrenos tradicionalmente regidos por los adultos o tomar iniciativas a la hora de formular los propios mecanismos participativos, quienes siguen liderando en todos los aspectos clave de la vida en el centro. No obstante, entre las conclusiones de sus estudios, Horna (2006), Mason y Urquhart (2001), Shier (2001) y Treseder (1997) señalan que la distribución del poder es central para ir avanzando hacia la participación genuina.

Ahora bien, como es sabido, la participación genuina sólo puede tener lugar en un centro que se articula alrededor de objetivos democráticos, procesos democráticos y acciones democráticas. Esto conlleva un giro copernicano en las estructuras de poder y en la imagen del adulto, y aún más del docente, como autoridad incuestionable. Cuando se piensa en una escuela democrática, la participación infantil cobra pleno sentido, porque sin la voz de los niños y las niñas el centro no funcionaría. Feito (2009) opina que las escuelas democráticas cumplen, al menos, tres condiciones: practicar una educación de calidad para todos (inclusión y éxito); centrarse en el discente y no en el docente con un currículo que se plantea desde la horizontalidad (democratización del aprendizaje); y la gestión compartida entre los diferentes miembros de la comunidad (participación real).

La participación, sin embargo, no debe terminar a las puertas de la escuela y tanto niños y niñas como profesionales deben recibir formación para que sea una realidad. La agenda política, en el contexto próximo, debería incluir a los niños, niñas y jóvenes, saberlos escuchar y confiar en las soluciones que aportan. Obviamente, se necesitan estrategias de coordinación con las entidades del entorno y el establecimiento de variedad de formas y canales para que todos los niños y todas las niñas sin excepción puedan participar.

Finalmente, nos gustaría señalar que esta investigación está todavía en sus primeros momentos y únicamente se han podido avanzar algunos datos, si bien los resultados obtenidos hasta el momento ya ofrecen una panorámica general de cuáles son las modalidades de participación del alumnado en los centros educativos. Somos conscientes de que se trata de un estudio de aproximación inicial al contexto y que para posteriores análisis deberemos ampliar la muestra y considerar las valoraciones de directores y directoras de otros centros educativos, así como contemplar el estudio con la visión de otros agentes de la comunidad educativa, 
como el alumnado, el profesorado y las familias,desarrollando, así, un estudio más global y combinado para el análisis de la participación infantil en la escuela. Sin duda, una de las limitaciones de la investigación presentada es la consideración de únicamente la perspectiva de directores y directoras de determinados centros educativos, aquellos que han optado por responder y donde podrían tener mayor representación los que desarrollan prácticas democráticas. Desde esta perspectiva, este estudio ha de servir de base para futuras investigaciones en otras Comunidades Autónomas, donde además de investigar con muestras aleatorias se incluyan y analicen variables como tipología de centro, género, edad o formación recibida, entre otras, con respecto a los directores y las directora. Asimismo, se considerará triangular las fuentes incorporando las opiniones de familias, alumnado y equipos docentes.

\section{Referencias bibliográficas}

Alfageme, E., Cantos, R., y Martínez, M. (2003). De la participación al protagonismo infantil. Propuestas para la acción. Madrid: Plataforma de Organizaciones de la Infancia.

Arnstein, S. (1969). A ladder of citizen participation, American Institute of Planners Journal, 35, 216-224.

Chawla, L. (2001). Evaluating children's participation: Seeking areas of consensus. PLA Notes, 42, 9-13.

Child watch International Research Network. (2006). Understanding and contextualizing children's real participation. A submission by Childwatch International Research Network to the Committee on the Rights of the Child Day of General Discussion September 2006.

Consejo de Europa. (2012). Council of Europe Recommendation on the participation of children and young people under the age of 18. Recuperado de https://rm.coe. int/CoERMPublicCommonSearchServices/DisplayDCTMContent?documen$\mathrm{tld}=090000168046 \mathrm{c} 478$

Cussiánovich, A. (2003). Historia del pensamiento social sobre la infancia. Universidad Nacional Mayor de San Marcos. Lima: Fondo Editorial de la Facultad de Ciencias Sociales.

Cussiánovich, A. (2010). Ensayos sobre infancia. Sujeto de derechos y protagonista. Lima: IFEJANT.

Cussiánovich, A. y Figueroa, E. (2009). Participación protagónica: ¿Ideología o cambio de paradigma? En M. Liebel y M. Martínez Muñoz (Coords.), Infancia y Derechos Humanos: Hacia una ciudadanía participante y protagónica (83-99).Lima: IFEJANT.

Departament d'Educació. Generalitat de Catalunya. (2007). La convivència en els centres d'educació infantil i primària. Barcelona: Generalitat de Catalunya. 
La dirección escolar ante la participación del alumnado

laura García-Raga, Maria Carme Boqué Torremorell y Montserrat Alguacil de Nicolás

Feito, R. (2009). Escuelas democráticas. RASE. Revista de la Asociación de Sociología de la Educación, 2(1), 17-33.

Francis, M. y Lorenzo, R. (2002). Seven realms of children's participation. Journal of Environmental Psychology, 22, 157-69.

Franklin, B. (1995). Handbook of Children's Rights. Londres: Routledge.

Gaitán, L. (2006). La nueva sociología de la infancia. Aportaciones de una mirada distinta. Revista Política y Sociedad, 43(1), 9-26.

Gaitán. L. (2008). Cambios en la infancia durante las últimas décadas. En AA.VV., V Encuentro la Ciudad de los Niños. La Infancia y la Ciudad: Una relación difícil (38-61). Getafe (Madrid): Acción Educativa.

Gal, T. (2017). An ecological model of child and youth participation. Children and Youth Services Review, 79,57-64. En https://doi.org/10.1016/j.childyouth.2017.05.029

Guichot, V. (2013). Participación, ciudadanía activa y educación. Teoría de la Educación, 25(2),25-47.

Hart, R. (1993). La participación de los niños. De la participación simbólica a la participación auténtica. Ensayos Innocenti. UNICEF.

Herbots, K. y Put, J. (2015). The participation disc. The International Journal of Children's Rights, 23(1), 154-188

Horizonte 2020. (s.f)Recuperado de http://eshorizonte2020.es/retos-sociales/europa-enun-mundo-cambiante-sociedades-inclusivas-innovadoras-y-reflexivas

Horna, P. (2006). Del dicho al hecho. Participación de niños/as y adolescentes en eventos con adultos. Suecia: Save the Children.

Howard, R., y Wieczorek, B. (2011). How to involve children and young people in school governance. Londres: Participation.

Kränzl-Nagl, R., yZartler, U. (2010). Children's participation in school and community: European perspectives. En B. Percy-Smith y N. Thomas, A Handbook of children and young people's participation. Perspectives from the ory and practice (164-173). Nueva York: Routledge.

Lansdown, G.(2001). Promoting children's participation in democratic decision-making. Siena: UNICEF Innocenti Research Centre, Florencia, Italia.

Liebel, M. (2000). La otra infancia. Niñez trabajadora y acción social. Lima: IFEJANT.

Liebel, M (2007). Entre protección y emancipación. Derechos de la Infancia y Políticas Sociales. Madrid: Facultad de Ciencias Políticas y Sociología. Departamento de Sociología II, Ecología Humana y Población.

Liebel, M. (2013). Niñez y Justicia Social. Repensando sus derechos. Santiago de Chile: Pehuén.

Lundy, L. (2007) Voice is not enough: Conceptualizing Article 12 of the UNCRC, British Educational Research Journal, 33(6),927-42. En http://dx.doi. org/10.1080/01411920701657033

Maranzana, L. y Gómez-Granell, C. (2011). Participació infantil i juvenil a la ciutat de Barcelona. Barcelona: Ayuntamiento de Barcelona (CIIMU).

Mason, J. y Urquhart, R. (2001). Confronting the Dilemmas of Involving Children as Partners: Collaborative Research Project Around Decision Making. Children Australia, 26(4), 16-21. 
Mitra, D.L. (2007). Student voice in school reform: From listening to leadership. En D. Thiessen y A. Cook-Sather (Eds.), International handbook of student experience in elementary and secondary school (727-744). Berlín: Springer.

Nolas, S. M. (2015). Children's participation, childhood publics and social change: A review. Children\&Society, 29(2), 157-167.

Novella, A. (2008). Formes de participació infantil: La concreció d'undret, Educació social, 38, 77-93.

Reddy, N. y Ratna, K. (2002). A journey in children's participation. Vimanapura: The Concerned for Working Children.

Santibáñez, D. (2008). Participación política y juventud: Limitaciones estructurales, incomunicabilidad y paradojas. Revista Observatorio de Juventud. Instituto Nacional de la Juventud, 5(19), 5-71.

Santibáñez, D., Delano, J. y Reyes, M. (2015). Participación y ejercicio de derechos. Santiago de Chile: Observatorio niñez y adolescencia.

SENAME. (2011). Informe resultados nacionales. Primera consulta nacional a niños, niñas y adolescentes de centros residenciales: "Mi derecho a ser escuchado" en el marco del Plan del Fortalecimiento del Buen Trato Institucional. Departamento de Protección de Derechos. Recuperado de http://ciperchile.cl/pdfs/07-2013/sename3/ Informe_mi_derecho_a_ser_escuchado.pdf

Shier, H. (2001). Pathways to participation: Openings, opportunities and obligations. Children \& Society, 15, 107-17.

Thomson, P. y Holdsworth, R. (2003). The orizing change in the educational 'field': Re-readings of 'student participation' projects'. International Journal of Leadership in Education, 6(4), 371-391.

Tisdall, E. K. M. y Punch, S. (2012). Not so 'new'? Looking critically at childhood studies. Children's Geographies, 10(3), 249-264.

Treseder, P. (1997). Empowering children and young people: Training manual. Londres: Save the Children and Children's Rights Office.

Trilla, J. y Novella, A. (2001). Educación y participación social de la infancia. Revista Iberoamericana de Educación, 26,137-164.

UNICEF. (2003). The state of the world's children 2003. Recuperado de http://www. UNICEF.org/sowc/archive/ENGLISH/The\%20State\%20of\%20the \%20World's\%20 Children\%202003.pdf

Woodhouse, B.B. (2003). Enhancing children's participation in policy formation. Arizona Law Review, 45,751-763. 
